\title{
Canadian guidelines for the management of asymptomatic microscopic hematuria in adults
}

\author{
Tim Wollin, MD, FRCSC; ${ }^{*}$ Bruno Laroche, MD; ${ }^{\dagger}$ Karen Psooy, MD $^{\ddagger}$
}

Can Urol Assoc J 2009;3(1):77-80

\section{Introduction}

Microscopic hematuria is a frequent reason for referral to urology. It is often found incidentally as a result of routine examination in patients without urinary tract symptoms. Although there is generally no debate about the need to fully investigate patients with gross hematuria, there is often controversy regarding the approach to the patient with asymptomatic microscopic hematuria. The main issues disputed are related to the detection and definition of significant microscopic hematuria, which patients should be investigated, and how should they be evaluated.

In 1998, the Canadian Urological Association published patient guidelines for asymptomatic hematuria. ${ }^{1} \mathrm{~A}$ decade has passed since the initial development of these principles. Thus, the CUA Patient Guidelines Committee has been given the mandate from the CUA to update the asymptomatic microscopic hematuria patient guidelines.

\section{Methods}

A review of the literature was performed for publication years 1998 to 2008 using MEDLINE, limited to the English language. A review of the American Urological Association Best Practice Policy Panel on Asymptomatic Microhematuria was also undertaken., ${ }^{2,3}$

The following principles and issues concerning the diagnosis and management of the patient with asymptomatic microscopic hematuria (AMH) are reviewed:

1. Definition of $\mathrm{AMH}$

2. Determination of which patients with AMH warrant further evaluation by a urologist a. Indications for nephrological assessment b. Indications for urological assessment

3. Urological evaluation of patients with $\mathrm{AMH}$

4. Follow-up of the patient with benign $\mathrm{AMH}$

The revised guideline was reviewed and critiqued by the Guidelines Committee. Furthermore, due to the lack of evidence especially as it relates to which patients should be evaluated, a consensus opinion was developed based on an informal survey of Canadian urologists from across the country and a final algorithm was designed (Fig. 1).

\section{Definition of microscopic hematuria}

In the 1998 AMH guideline, significant microscopic hematuria was defined as greater than 2 RBCs/hpf on two microscopic urinalysis without recent exercise, menses, sexual activity or instrumentation. Review of the literature did not reveal any evidence to warrant changing this definition.

\section{Which patients require evaluation for AMH?}

Once microscopic hematuria has been confirmed, which patients require further evaluation? First, in patients with a history of recent exercise, menses, sexual activity or urethral trauma/instrumentation, a repeat microscopic exam should be done once the contributing factor has ceased. ${ }^{3}$ If the subsequent exam is negative, then further work-up is not required.

Next, it should be determined if the patient's hematuria could be secondary to a glomerular cause. The presence of proteinuria, red cell casts, or dysmorphic red blood cells on microscopic exam and/or an elevated creatinine is suggestive of a glomerular cause of hematuria and these patients should be referred to a nephrologist for further investigation. ${ }^{3-5}$ Further urological work-up including cystoscopy may not be required in this situation. All other patients should be assessed for the need of further evaluation.

\section{Evaluation of the upper and lower urinary tracts}

There is inadequate evidence in the literature 
Wollin et al.

to definitively recommend which patients should undergo full or partial urological evaluation for their microscopic hematuria. The AUA Best Policy Panel recommends that all patients with microscopic hematuria undergo evaluation of the upper tracts while only high-risk patients have lower tract evaluation..$^{2,3}$ Conversely, guidelines out of the UK endorse evaluation of the upper and lower urinary tracts for all patients with no risk stratification. ${ }^{6,7}$ The 1998 CUA guideline advocated evaluation of all patients over the age of 40 years only. ${ }^{1}$

The upper urinary tract is evaluated with diagnostic imaging. The aim of imaging is to detect neoplasms, urolithiasis, and obstructive or inflammatory lesions. Intravenous urography (IVU), ultrasonography (US), and enhanced computed tomography (CT) are the modalities used most commonly. Although there are numerous studies describing the application of these modalities in this setting, ${ }^{8-11}$ there are no comparative studies that can help establish an evidence-based policy.

Intravenous urography (IVU) is the study that has been traditionally used to investigate the upper urinary tract. It is widely available, easy to perform, and is able to detect transitional cell carcinoma of the upper urinary tract with an acceptable degree of sensitivity. However, fewer centres are offering IVU and the study

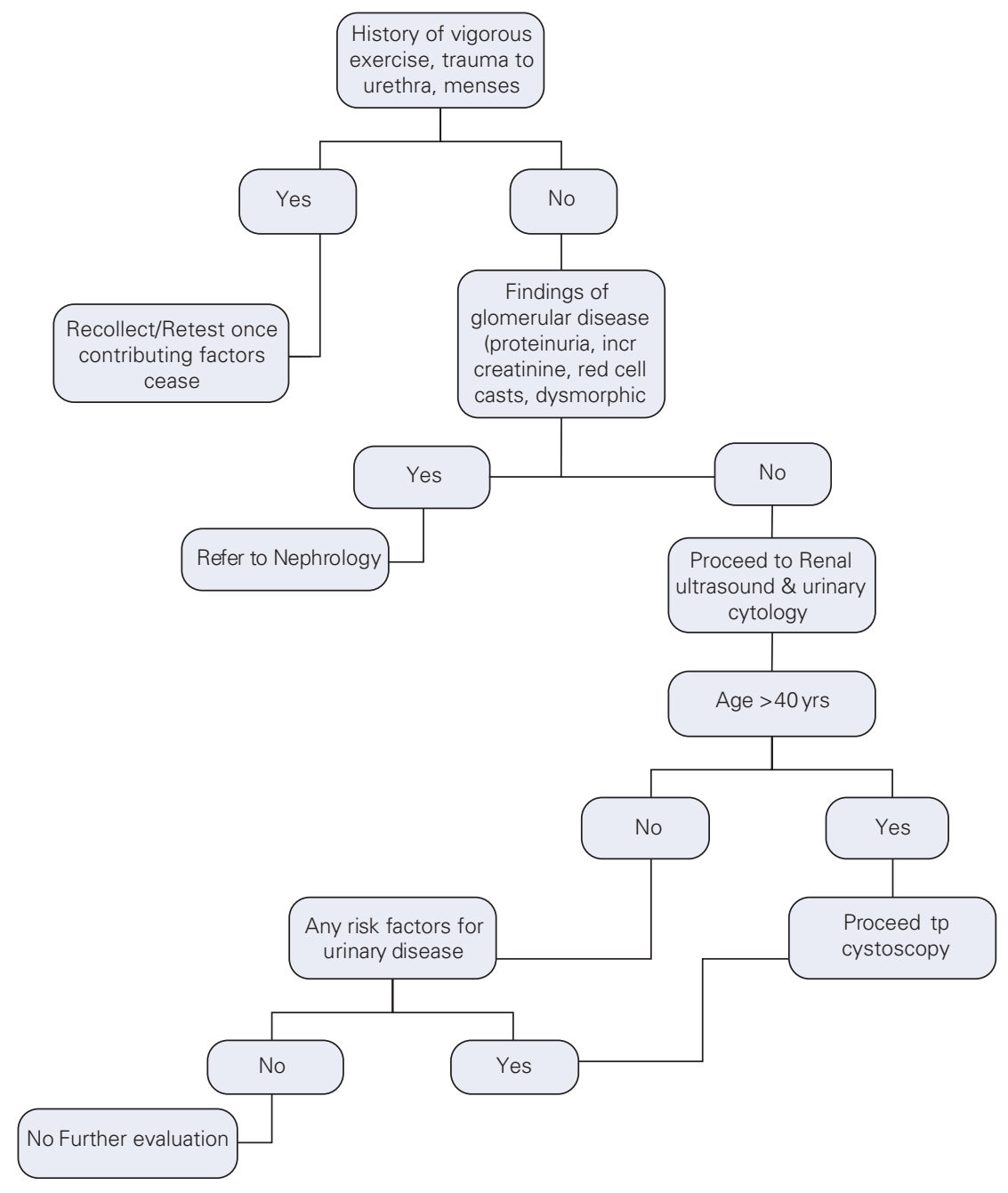

Fig. 1. Algorithm for the evaluation of the adult patient with asymptomatic microscopic hematuria. 
has a limited sensitivity for diagnosing renal cell carcinoma. ${ }^{11}$

Ultrasound is also widely available. Moreover, it is noninvasive, does not require ionizing radiation or intravenous contrast and, compared to CT, is less expensive. It is superior to IVU for evaluating the renal parenchyma and renal cysts. However, like IVU, it has limited sensitivity for small renal masses..$^{12}$ It also has a limited sensitivity for diagnosing transitional cell carcinoma.

$\mathrm{CT}$ is the optimal test for examining the renal parenchyma for masses. It is also the best test for diagnosing renal/ureteral calculi and renal and/or peri-renal infections. The disadvantages of CT include decreased availability in some centres, the need for ionizing radiation, exposure to intravenous contrast, and increase expense compared to IVU and US.

Due to the lack of evidence in this setting, any of these imaging modalities are acceptable for the evaluation of the patient with microscopic hematuria. However, taking patient safety (ionizing radiation and exposure to i.v. contrast), availability, and cost into consideration, it is recommended that US be used as the imaging test of first choice. CT and IVU are justified when additional tests are believed to be indicated.

The lower urinary tract is evaluated with urinary cytology and cystoscopy. Since cytology is an easy test to perform, is noninvasive and is relatively inexpensive, it is a recommended investigation for lower tract assessment that should be obtained early on in the evaluation of the patient with microscopic hematuria. The sensitivity for detecting transitional cell carcinoma is moderate and ranges from $40 \%$ to $75 \%$ depending on the number and type of urine specimens examined, the grade of the tumour, and the expertise of the cytopathologist. ${ }^{3}$

The 1998 CUA guidelines state

patients over the age of 40 have a significant incidence of pathology such as transitional cell carcinoma and renal cell carcinoma. These lesions are rare in patients under the age of 40. Therefore cystoscopy of young patients with asymptomatic microscopic hematuria is not warranted..$^{13-16}$

The literature still supports this principle. ${ }^{3,4,17}$ However, in addition to an age over 40 years, there are other risk factors that should be considered sig- nificant for disease in patients with microscopic hematuria: ${ }^{3,18}$

- Smoking history

- Occupational exposure to chemicals or dyes (benzenes, aromatic amines)

- History of irritative voiding symptoms

- Analgesic abuse with phenacitin

- History of pelvic irradiation

- Cyclophosphamide exposure

Therefore, patients that are under the age of 40 years with none of the above risk factors do not require cystoscopy. Conversely, all patients over the age of 40 years need cystoscopy. Any patient with positive or atypical cytology, or those with one or more risk factors also need cystoscopic evaluation.

\section{Recommended follow-up of the patient with AMH}

Following a negative evaluation for $\mathrm{AMH}$, between $1 \%$ and $3 \%$ of patients have been reported to be diagnosed with a urological malignancy within three years. ${ }^{16,19,20} \mathrm{~A}$ small proportion may also develop renal insufficiency due to glomerular disease. ${ }^{21-23} \mathrm{As}$ a result, some form of patient follow-up is recommended after negative initial evaluation for $\mathrm{AMH}$.

An evidence-based policy cannot be recommended at this time; however an approach similar to that recommended by the AUA Best Policy Panel is acceptable. Patients should be followed by their primary care physician with urinalysis, urinary cytology, and blood pressure checks at 6, 12, 24 and 36 months., ${ }^{3,18}$ If a patient develops gross hematuria, positive or atypical cytology, or irritative voiding symptoms without infection, then repeat urological evaluation is advised. The development of hypertension, proteinuria, or the finding of glomerular bleeding would necessitate referral to a nephrologist. If none of these occur after three years, then routine follow-up for persistent hematuria can be ceased.

\section{Guideline summary}

1. Definition of microscopic hematuria:

a. Greater than 2 RBCs/hpf on two microscopic urinalysis without recent exercise, menses, sexual activity or instrumentation (Grade C Recommendation; Level of Evidence $3 \mathrm{~b}$ and 4).

2. Indications for nephrology referral:

a. The presence of proteinuria, red cell casts, 
Wollin et al.

or dysmorphic red blood cells on microscopic exam and/or an elevated creatinine is suggestive of a glomerular cause of hematuria (Grade C Recommendation; Level 3b and 4).

3. Indications and extent of urological evaluation:

a. All patients with microscopic hematuria should be investigated by urine cytology and upper tract imaging (Grade D Recommendation; Level 5 evidence).

b. Patients $>40$ years of age, those with positive or atypical cytology, or any patient with the presence of any of the following risk factors should have their lower tract assessed by cystoscopy (smoking history, occupational exposure to chemicals or dyes, history of irritative voiding symptoms, analgesic abuse with phenacitin, history of pelvic irradiation, or cyclophosphamide exposure) (Grade C Recommendation; Level $3 \mathrm{~b}$ and 4 evidence).

4. Upper tract evaluation:

a. There is limited evidence to strongly recommend one modality. Thus, although ultrasound, CT or IVP are acceptable, taking patient safety (ionizing radiation and exposure to i.v. contrast), availability, and cost into consideration, it is recommended that ultrasound be used as the imaging test of first choice (Grade C Recommendation; Level 3b and 4 evidence).

5. Follow-up after negative evaluation:

a. Patients should be followed by their primary care physician with urinalysis, urinary cytology, and blood pressure checks at 6, 12, 24 and 36 months (Grade C Recommendation; Level $3 \mathrm{~b}$ and 4 evidence).

i. Repeat urological assessment is required if a patient develops gross hematuria, positive or atypical cytology, or irritative voiding symptoms without infection.

ii. Nephrology referral is indicated with the development of hypertension, proteinuria, or the finding of glomerular bleeding.

iii. If none of these occur after three years, then routine follow-up for persistent hematuria can be ceased.

From the *Division of Urology, University of Alberta, Edmonton, Atla., †SaintFrançois d'Assise Hospital, Centre hospitalier univérsitaire de Québec, Québec City, Que., and the fDivision of Pediatric Urology, Winnipeg Children's Hospital, Winnipeg, Man.

This article has been peer reviewed.
Competing interests: None declared.

\section{References}

1. Canadian Urological Association. Asymptomatic microscopic hematuria. 1998.

2. Grossfeld GD, Litwin MS, Wolf IS, et al. Evaluation of asymptomatic microscopic hematuria in adults: the American Urological Association best practice policy - part I: definition, detection, prevalence, and etiology. Urology 2001;57:599-603.

3. Grossfeld GD, Litwin MS, Wolf JS, et al. Evaluation of asymptomatic microscopic hematuria in adults: the American Urological Association best practice policy — part Il: patient evaluation, cytology, voided markers, imaging, cystoscopy, nephrology evaluation, and follow-up. Urology 2001;57:604-10.

4. Tomson C, Porter T. Asymptomatic microscopic or dipstick haematuria in adults: which investigations for which patients? A review of the evidence. BJU Int 2002;90:185-98.

5. Savige J, Buzza M, Dagher H. Haematuria in asymptomatic individuals. BMJ 2001; 322:942-3.

6. Scottish Intercollegiate Guidelin Network. Investigation of asymptomatic microscopic haematuria in adults. SIGN Publication (17). 1997. Edinburgh.

7. Finlayson JA. Asymptomatic haematuria. Scottish guidelines are different from authors interpretation of best available evidence. [author reply 1599-600]. BMJ 2000;320:1598.

8. McNicholas MM, Raptopoulos VD, Schwartz RK, et al. Excretory phase CT urography for opacification of the urinary collecting system. AIR Am J Roentgenol 1998;170:1261-7.

9. Igarashi T, Muakami S, Shichijo Y, et al. Clinical and radiological aspects of infiltrating transitional cell carcinoma of the kidney. Urol Int 1994;52:181-4.

10. Buckley JA, Urban BA, Soyer P, et al. Transitional cell carcinoma of the renal pelvis: a retrospective look at CT staging with pathologic correlation. Radiology 1996;201:194-8.

11. Warshaver DM, MCCarthy SM, Street L, et al. Detection of renal masses: sensitivities and specificities of excretory urography/linear tomography, US, and CT. Radiology 1988; 169:363-5.

12. Jamis-Dow CA, Choyke PL, Jennings SB, et al. Small ( $<$ or $=3-\mathrm{cm})$ renal masses: detection with CT versus US and pathologic correlation. Radiology 1996;198:785-8.

13. Greene LF, O'Shaughnessy EJ Jr, Hendricks ED. Study of five hundred patients with asymptomatic microhematuria. J Am Med Assoc 1956;161:610-3.

14. Bard RH. The significance of asymptomatic microhematuria in women and its economic implications. A ten-year study. Arch Intern Med 1988; 148:2629-32.

15. Thompson IM. The evaluation of microscopic hematuria: a population-based study. J Urol 1987;138:1 189-90.

16. Murakami S, Igarashi T, Hara S, et al. Strategies for asymptomatic microscopic hematuria: a prospective study of 1,034 patients. I Urol 1990;144:99-101.

17. Suzuki Y, Sasagawa I, Abe Y, et al. Indication of cystoscopy in patients with asymptomatic microscopic haematuria. Scand I Urol Nephrol 2000;34:51-4.

18. Grossfeld GD, Wolf JS Jr, Litwan MS, et al. Asymptomatic microscopic hematuria in adults: summary of the AUA best practice policy recommendations. Am Fam Physician 2001;63:1145-54

19. Golin AL, Howard RS. Asymptomatic microscopic hematuria. J Urol 1980;124:389-91.

20. Carson CC III. III, Segura JW, Greene LF. Clinical importance of microhematuria. JAMA 1979;241:149-50.

21. Dische FE, Weston MJ, Parsons V. Abnormally thin glomerular basement membranes associated with hematuria, proteinuria or renal failure in adults. Am J Nephrol 1985;5:103-9.

22. Goel S, Davenport A, Goode NP, et al. Clinical features and outcome of patients with thin and ultrathin glomerular membranes. QJM 1995;88:785-93.

23. Nieuwhof $C M$, de Heer $F$, de Leeuw $P$, et al. Thin GBM nephropathy: premature glomerular obsolescence is associated with hypertension and late onset renal failure. Kidney Int 1997:51:1596-601.

Correspondence: Dr. Tim Wollin, Division of Urology, University of Alberta, Alberta Urology Institute Inc., Suite 400 Hys Centre, 11010-101 St., Edmonton AB T5H 4B9; twollin@ualberta.ca 